\section{Quality of Care for Older Patients With Non-Cancer Diagnoses Under the End-of-Life Care Program}

\author{
Tang Wing-Hang', Ma Hon-Ming', Lee Jenny SW'1, Hui Elsie ${ }^{2,3}$, Woo Jean ${ }^{4}$ and Kwan \\ Joseph SK $^{5^{*}}$
}

${ }^{1}$ Honorary Clinical Assistant Professor, Department of Medicine and Therapeutics, Prince of Wales Hospital, The Chinese University of Hong Kong, Hong Kong, China

${ }^{2}$ Honorary Clinical Associate Professor, Department of Medicine and Therapeutics, Prince of Wales Hospital, The Chinese University of Hong Kong, Hong Kong, China

${ }^{3}$ Chief-of-Service, Department of Medicine and Geriatrics, Shatin Hospital, Hong Kong, China

${ }^{4}$ Chairman, Department of Medicine and Therapeutics, Prince of Wales Hospital, The Chinese University of Hong Kong, Hong Kong, China

${ }^{5}$ Clinical Associate Professor, Department of Medicine, The University of Hong Kong, Hong Kong, China

\section{Citation}

Wing-Hang T, Hon-Ming M, Jenny SW L, Elsie H, Jean W, Joseph SK K. Quality of care for older patients with non-cancer diagnoses under the end-of-life care program. Palliat Med Hosp Care Open J. 2015; 2(1): 7-13. doi: 10.17140/PMHCOJ-2-111

\section{Copyright}

(C2015 Joseph SK K. This is an open access article distributed under the Creative Commons Attribution 4.0 International License (CC BY 4.0), which permits unrestricted use, distribution, and reproduction in any medium, provided the original work is properly cited.

\section{ABSTRACT}

Background: End-of-life (EOL) care is an important part of geriatric medicine in view of rapidly ageing populations in the world.

Aim: We aimed to evaluate the quality of care for older patients with non-cancer terminal illnesses, who died in 2010, under the EOL care program of an academic medical unit in Hong Kong. This unit consisted of an acute hospital, Prince of Wales Hospital (PWH) and a convalescence hospital (Shatin Hospital, SH).

Methods: This was a retrospective hospital-based audit of clinical effectiveness of the EOL service. We reviewed the quality of patient care during the final seven days of life. The quality of care was evaluated based on the compliance rates of five selected goals and the adoption of futile life-sustaining procedures and treatments.

Results: Case records of 129 patients in the EOL care program were analyzed. Two goals, including minimization of regular monitoring of vital signs and no blood taking, achieved over $70 \%$ compliance at $\mathrm{SH}$ and $0 \%$ at $\mathrm{PWH}$. The compliance rates of discontinuation of non-essential medications were $46.4 \%$ in SH and $47.1 \%$ in $\mathrm{PWH}$; and the compliance rates of switching essential medications to non-oral routes were $63.4 \%$ in SH and $70.6 \%$ in PWH (not statistically significant). The compliance rates of using as-required intravenous or subcutaneous medications were extremely low $(<2 \%)$ at both hospitals. All futile life-sustaining procedures and treatments were initiated at the PWH.

Conclusions: We demonstrated significant differences in the quality of EOL care between the acute hospital and convalescence hospital. Greater emphasis on specialist training and education with allocation of resources may improve the EOL care in both settings.

KEYWORDS: End of life; Elderly; Chronic diseases; Advanced care plan; Palliative care.

ABBREVIATIONS: EOL: End-of-life; PWH: Prince of Wales Hospital; SH: Shatin Hospital; ACP: Advanced Care Plan; CHF: Congestive Heart Failure; COPD: Chronic Obstructive Pulmonary Disease; ESRF: End-stage renal failure; DNR: Do Not Resuscitation; AED: Accident and Emergency Department; LCP: Liverpool Care Pathway; WHO: World Health Organization; CPR: Cardiopulmonary resuscitation; AMT: Abbreviated Mental Test; OGD: Oesophagogastro-duodenoscopy; LCP: Liverpool Care Pathway; ACP: Advanced Care Planning. 


\section{INTRODUCTION}

End of life (EOL) care is increasingly recognized as an essential component of healthcare system, but the access to EOL care service is far from satisfying the demand, particularly with an aging population in the world. ${ }^{1}$ It was also shown that older in-patients were more likely to suffer from inappropriate pain control and excessive interventions at the terminal stage of their lives. ${ }^{2}$ Therefore, it is important to deliver high quality EOL care for frail older patients by setting up an Advanced Care Plan (ACP), offering adequate pain and symptom control without intention to hasten or postpone death, relieving caregivers' burden and strengthening rapport with the relatives. ${ }^{3-6}$ In Hong Kong, people usually died in the hospital instead of at home or in the residential home in view of social culture and legislations. Most of the EOL care was therefore conveyed through the hospital setting. However, previous studies of EOL care have reported dissatisfaction from patients and their family members about symptom control and failure to address their physical and psychosocial needs in the hospital setting. ${ }^{7-10}$ The aim of the study reported in this paper is to evaluate and compare the quality of EOL care for older patients with non-cancer diagnoses, in the acute and convalescence hospitals, by reviewing their clinical records during the last seven days of life.

\section{EOL Care in Shatin Hospital, Hong Kong}

With a capacity of 300 medical beds, shatin hospital (SH) offers a sub-acute, convalescence and step-down care for patients transferred from the prince of wales hospital (PWH), which provides acute general medical services in New Territories East region with 0.7 million populations of which $12 \%$ are people aged over 65 . In order to improve the quality of EOL care for non-cancer older patients with chronic illness, SH medical unit launched an EOL care program in 2008 for those with non-cancer chronic illnesses including Congestive Heart Failure (CHF), Chronic Obstructive Pulmonary Disease (COPD), Endstage renal failure (ESRF), stroke, dementia, other neurodegenerative diseases, and frailty who approach their end stage of life. The focus of this program was to palliate symptoms, enhance patient and family satisfaction with palliative care services, and allow the patient to rest in his home environment for as long as possible during the final phase of life. There were three commencing criteria:

1. Patient and relative not opting for active treatment; and

2. Existing Do Not Resuscitation (DNR) order; and

3. Satisfying the prognostic indicators of advanced disease of chronic illnesses.

After identifying a potential case for the EOL care program, the medical team in SH explain the program details to the patient and the family, and invite the patient to join the program by establishing an ACP and the DNR order. If the patient developed clinical deterioration, geriatricians might arrange direct clinical admission to $\mathrm{SH}$ for him to receive EOL care in their terminal stage of life. The patient would still be managed by the general medical team as there was no special palliative team in $\mathrm{SH}$ for caring the EOL cases. However, if the patient was critically ill, given that SH did not have Accident and Emergency Department (AED), the patient was inevitably sent to AED in other hospitals, most likely to $\mathrm{PWH}$, for immediate treatment, because ambulance paramedics have to transport emergency patients to the nearest AED according to the government guideline. The patient might subsequently be resuscitated in AED and admitted to acute medical units in PWH for further care.

Another similar project was undertaken in the oncology unit of another regional hospital in Hong Kong from July to September 2009 on the use of integrated care pathway for EOL patients with cancer. The compliance rates of the following six selected items ranged from $80-100 \%$, including: 1) discontinuation of non-essential medications; 2) switching of essential medications to non-oral routes; 3 ) prescription of as-required intravenous or subcutaneous palliative medications; 4) minimization of regular monitoring of vital signs; 5) practice of flexible visiting hours; and 6) order of 'no further investigation'.

In Hong Kong, there was a lack of data on the compliance of EOL care pathway in acute $v s$. convalescence medical units. A typical example would be the use of unnecessary lifesustaining procedures or treatments for older patients with terminal non-cancer diseases despite their wishes for comfort care. ${ }^{10-12}$

\section{METHODS}

\section{Method}

This was a retrospective hospital-based audit of clinical effectiveness of the EOL service in SH and PWH. We collected routine clinical data from the case notes and clinical management system to review and compare the quality of EOL care provided during the final seven days of life for patients in $\mathrm{SH}$ and PWH. Since this was a retrospective audit with no novel intervention or collection of identifiable data, our study has the necessary ethical approval

\section{Patient Recruitment}

We retrospectively reviewed the clinical records of all the patients of EOL care program who died in 2010. Their socio-demographic characteristics, residential status, diagnoses, comorbidities, clinical characteristics (functional status, cognition, long-term use of naso-gastric feeding, urinary catheter and oxygen therapy), which reflected their background functional status, were recorded and summarized.

\section{Evaluation Criteria of the Quality of EOL Care}

The quality of EOL care was assessed, based on: 1) the 
compliance rates of five selected goals (see below), and 2) use of futile life-sustaining procedures and treatment in the last seven days of life. The five selected goals included: 1) discontinuation of non-essential medications, 2) minimization of regular monitoring of vital signs, 3) no blood-taking, 4) use of as-required intravenous or subcutaneous palliative medications, and 5) switching of essential medications to non-oral routes. These goals were adapted from the Liverpool Care Pathway (LCP) and the EOL care pathway used in the palliative care unit in Hong Kong, China. The compliance rate of each goal was calculated as the percentage of patients with goals achieved, with the gold standard being $100 \%$ compliance.

Regarding the first goal, the World Health Organization (WHO) recommended a list of essential medications for use in palliative care in 2013, including morphine, ibuprofen, amitriptyline, fluoxetine, diazepam, loperamide, lorazepam, haloperidol, metoclopramide, dexamethasone, hyoscine hydrobromide, docusate sodium, senna and lactulose..$^{13}$ In this study, medications in the same group of those listed above were classified as essential medications for symptom control in the dying phase. On the contrary, continuation of anti-hypertensive drugs, antiplatelet drugs, glucose-lowering drugs, and mineral and electrolyte supplements in the last day of life was likely categorized as non-essential, but it should be reviewed case by case.

For the fifth goal, prescription of essential medications was reviewed on the last day instead of the final seven days because patients might be able to eat initially and oral administration of essential medications could be clinically appropriate then. Administration of medications via nasogastric or gastrostomy tube was considered appropriate in tube-fed patients.

\section{Use of Futile Life-Sustaining Procedures and Treatments}

Use of futile life-sustaining procedures and treatments during the last seven days of life included 1) Cardiopulmonary resuscitation (CPR); 2) non-invasive and mechanical ventilation; 3) use of inotropic agents and fluid resuscitation for hypotension or shock, and 4) the use of invasive diagnostic procedures. In Hong Kong, intravenous or subcutaneous hydration is commonly continued until death due to cultural reasons and family wishes, so this has not been counted as life-sustaining treatment in this audit.

\section{Statistical Analysis}

Categorical variables were presented as counts and percentages, and continuous variables as mean values with standard deviations. The compliance rates of five selected goals in $\mathrm{SH}$ and PWH were compared and tested if there was a statistically significant difference between two hospitals by using two samples z-test.

\section{RESULTS}

\section{Clinical characteristics of EOL patients}

Table 1 summarizes the clinical characteristics of 146 patients included in this audit. Over half $(54.8 \%)$ of them were residing in nursing homes, and the mean duration of institutionalization was 4.2 years. Stroke, which caused significant disability and need for naso-gastric tube feeding, was the most common indication for EOL care (26\%), followed by dementia (17.1\%), chronic renal failure (15.8\%), COPD (11.6\%), congestive heart

\begin{tabular}{|l|c|}
\hline Demographics & $\mathbf{n}(\%)$ unless otherwise stated \\
\hline Mean (SD) Age, years & $83.4( \pm 9.4)$ \\
\hline Gender, male & $66(45.2 \%)$ \\
\hline Nursing home residents & $80(54.8 \%)$ \\
\hline Community-dwelling & $66(45.2 \%)$ \\
\hline Lived with children & $30(45.5 \%)$ \\
\hline Lived with spouses & $29(43.9 \%)$ \\
\hline Lived with other relatives & $3(2.1 \%)$ \\
\hline Lived alone & $4(6.1 \%)$ \\
\hline Availability of domestic helper & $20(30.3 \%)$ \\
\hline & \\
\hline Main Diagnosis for EOL Care & \\
\hline Stroke & $38(26.0 \%)$ \\
\hline Advanced dementia & $25(17.1 \%)$ \\
\hline Chronic renal failure & $23(15.8 \%)$ \\
\hline Chronic obstructive pulmonary disease & $17(11.6 \%)$ \\
\hline Congestive heart failure & $15(10.3 \%)$ \\
\hline Frailty & $14(9.6 \%)$ \\
\hline Chronic liver disease & $10(6.8 \%)$ \\
\hline
\end{tabular}




\begin{tabular}{|c|c|}
\hline Others & $4(2.7 \%)$ \\
\hline \multicolumn{2}{|l|}{ Comorbidities } \\
\hline Mean number of comorbidities & 5.4 \\
\hline Hypertension & $93(63.7 \%)$ \\
\hline Pressure ulcer & $83(56.8 \%)$ \\
\hline Cognitive impairment & $76(52.1 \%)$ \\
\hline Stroke & $71(48.6 \%)$ \\
\hline Anemia & $62(42.5 \%)$ \\
\hline Diabetes mellitus & $53(37.0 \%)$ \\
\hline Congestive heart failure & $43(29.5 \%)$ \\
\hline End-stage renal failure & $41(28.1 \%)$ \\
\hline Atrial fibrillation & $32(21.9 \%)$ \\
\hline Chronic obstructive pulmonary disease & $30(20.5 \%)$ \\
\hline Hip fracture & $21(14.4 \%)$ \\
\hline \multicolumn{2}{|l|}{ Clinical Characteristics } \\
\hline Mean (SD) Abbreviated Mental Test score (max 10$)$ & $2.2( \pm 3.3)$ \\
\hline Mean (SD) Norton Risk for pressure sore $(\max 30)$ & $10.8( \pm 3.7)$ \\
\hline Mean (range) Number of oral medications & $3(0-16)$ \\
\hline Mobility status: bed-ridden & $63(43.2 \%)$ \\
\hline Mobility status: chair-bound & $42(28.8 \%)$ \\
\hline Mobility status: walk with or without aids & $41(28.1 \%)$ \\
\hline Long-term naso-gastric feeding & $63(43.2 \%)$ \\
\hline Long-term urinary catheterization & $21(14.4 \%)$ \\
\hline Long-term oxygen therapy & $23(15.8 \%)$ \\
\hline
\end{tabular}

Table 1: Baseline characteristics of patients in this audit $(\mathrm{N}=146)$.

failure (10.3\%), frailty (9.6\%) and chronic liver diseases $(6.8 \%)$. Over $70 \%$ of the patients had very limited mobility (either chairbound or bedridden). 85 of the 146 patients $(58.2 \%)$ were verbally non-communicative. 139 of the 146 patients were assessed using the Abbreviated Mental Test (AMT) score, with the mean score of 2.2 (maximum score 10), of which 113 patients $(81.3 \%$ ) had a score equal to or lower than 5 . The mean body mass index was $18.2 \mathrm{~kg} / \mathrm{m}^{2}$ and mean serum albumin level was $27.8 \mathrm{~g} / \mathrm{l}$.

The median length of stay of the last episode of hospitalization was 17 days (range 1-193 days) at $\mathrm{SH}$, and 3 days (range 1-20 days) at PWH. 131 of the 146 patients (89.7\%) died within 6 months of being enrolled into the EOL care program. Nearly half of the patients $(71 / 146,48.6 \%)$ died during the same admission for which they were enrolled into the EOL care program. 114 patients died at SH, 19 at $\mathrm{PWH}, 5$ within the AED of PWH, and 8 at other hospitals.

\section{Quality of EOL Care}

To study the compliance rates of the five selected goals, case notes regarding the clinical conditions of patients in the last seven days of life were reviewed. However, 5 out of 146 patients were certified dead within the AED of PWH, 8 died in other hospitals, and case notes of 4 patients were missing, so only case notes of 129 patients could be retrieved and data ana- lyzed. Table 2 summarizes the compliance rates of five selected goals. Two goals, including minimization of regular monitoring of vital signs and no blood taking, achieved over $70 \%$ compliance at $\mathrm{SH}$, but $0 \%$ at $\mathrm{PWH}$. The compliance rates of discontinuation of non-essential medications in $\mathrm{SH}$ and $\mathrm{PWH}$ was $46.4 \%$ and $47.1 \%$ respectively without statistically significant difference $(\mathrm{p}=0.961)$. Over $90 \%$ of the patients continued with their anti-platelet or anticoagulant agents, over $80 \%$ with their antihypertensive drugs, and over $30 \%$ received antibiotics during the last day of life. For the switching of essential medications to non-oral routes, the compliance rate in $\mathrm{SH}(63.4 \%)$ was lower than that in PWH (70.6\%), but there was no statistically significant difference $(\mathrm{p}=0.564)$. The compliance rate of prescribing as-required intravenous or subcutaneous palliative medications was extremely low $(<2 \%)$ in both $\mathrm{PWH}$ and $\mathrm{SH}$.

\section{Use of Futile Life-Sustaining Procedures and Treatments}

Table 3 summarizes the number of futile life-sustaining procedures and treatments. All of these futile procedures and treatments were given in $\mathrm{PWH}$, and none in $\mathrm{SH}$.

\section{DISCUSSION}

In this study, we assessed and compared the quality of 


\begin{tabular}{|l|c|c|c|c|}
\hline & $\begin{array}{c}\text { Overall } \\
\mathbf{( N = 1 2 9 )}\end{array}$ & $\begin{array}{c}\text { SH } \\
(\mathbf{n = 1 1 2})\end{array}$ & $\begin{array}{c}\mathbf{P W H} \\
\mathbf{( n = 1 7 )}\end{array}$ & P-value \\
\hline Discontinuation of non-essential medications & $60(46.5 \%)$ & $52(46.4 \%)$ & $8(47.1 \%)$ & 1.000 \\
\hline Minimization of regular monitoring of vital signs & $81(62.8 \%)$ & $81(72.3 \%)$ & $0(0.0 \%)$ & $<0.001$ \\
\hline No blood taking & $87(67.4 \%)$ & $87(77.7 \%)$ & $0(0.0 \%)$ & $<0.001$ \\
\hline Use of as-required IV or SC palliative medications & $2(1.6 \%)$ & $2(1.8 \%)$ & $0(0.0 \%)$ & 1.000 \\
\hline Switching of essential medications to non-oral routes & $83(64.3 \%)$ & $71(63.4 \%)$ & $12(70.6 \%)$ & 0.787 \\
\hline
\end{tabular}

Table 2: Compliance rates of five selected goals.

\begin{tabular}{|l|c|}
\hline \multicolumn{1}{|c|}{ Example } & Number of cases \\
\hline Cardio-pulmonary resuscitation & 6 \\
\hline Use of inotropic drugs for treatment of shock & 3 \\
\hline Intubation & 2 \\
\hline Non-invasive or mechanical ventilation & 2 \\
\hline Fluid resuscitation for hypotension & 2 \\
\hline Use of diagnostic Oesophago-Gastro-Duodenoscopy & 1 \\
\hline Use of diagnostic abdominal paracentesis & 1 \\
\hline
\end{tabular}

Table 3: Examples of futile treatments in the last 7 days at $\mathrm{PWH}$.

EOL care for older patients with non-cancer diagnoses under the EOL care program in the convalescence hospital $v s$. acute hospital by the compliance rates of five criteria, and the use of futile procedures and treatments. The results highlighted service gaps and areas for improvement in the EOL care program, particularly within the acute hospital. The compliance rate of discontinuation of non-essential medications was lower than $50 \%$ at both hospitals. These figures suggested that medications were less likely to be titrated according to the individual clinical conditions towards the end of life. Compared with $100 \%$ compliance rate reported in a similar study in a local oncology unit, ${ }^{14}$ the lower compliance rate in our study might have resulted from insufficient guideline and staff awareness during initial assessment. Minimizing the drug load by signing off relatively nonessential medications should be emphasized for the sake of comfort care in any? medical unit.

It was also shown that essential medications for symptom control were less likely to be used at the acute hospital, for example, in the use of morphine. It was potentially associated with differences in the working culture between convalescence and acute hospitals. The concept of EOL care permeated throughout the convalescence unit of the convalescence hospital, so its clinical staff was better prepared to take care of the dying patients, especially in the aspect of symptom control.

Overall, the rate of using essential medications via nonoral routes in the last days of life was fair (63.4\%). However, the rate of prescribing as-required palliative medications via intravenous or subcutaneous route was low in both hospitals, below $2 \%$. It signified a potential increased risk of inadequate symptom control in the final phase of life. The frequency of vital sign monitoring was noted to be much higher at the acute hospital compared to convalescence hospital. It should be noted that frequent monitoring of vital signs may cause unnecessary dis- comfort, emotional arousal and anxiety among patients and their family members. Furthermore, any abnormal findings would lead to further excessive investigations, such as blood tests, Xrays and electrocardiograms. Clinical staff should shift their attention from routine monitoring of vital signs to focus on symptom control, such as dyspnoea, pain, fever and restlessness. A higher priority should be placed on routine nursing care, including mouth care, skin hygiene, turning and assisted oral intake.

Two of our study patients underwent invasive investigations and procedures for the workup of malignancy (Oesophago-gastro-duodenoscopy (OGD) and abdominal paracentesis)), despite the fact that they were unlikely to be a candidate for surgery, radiotherapy and chemotherapy, and their life expectancy was shorter than six months. Not only would the investigations not alter the treatment, they might also induce extra physical and psychological stress on the patients and their family members. Nonetheless, the DNR order was not equivalent to 'no-treatment' order, and it could be worthwhile ordering investigations but only if the focus was to improve the quality of life, such as blood transfusion for symptomatic anemia, or thoracentesis for symptomatic pleural effusion.

Despite being offered direct admission to the convalescence hospital when necessary, many patients were admitted to the acute hospital through the AED, because of acute medical deterioration. They often spent a few days at the acute hospital before being transferred to convalescence. As mentioned likewise in an article review, ${ }^{15}$ patients at the acute hospital were handled by many different physicians with variable clinical expertise in EOL or geriatric care. Interns were usually the first medical contact to deal with the symptoms reported by the nurses. It was not uncommon that some futile treatments were initiated by the interns or junior medical staff (e.g. as non-invasive ventilation, fluid resuscitation and ordering blood and imaging tests), although many of which were subsequently stopped by 
the attending physicians. These often happened during non-office hours or the middle of the night. Another major problem was a generally poor quality of documentation for decisions or treatment plans, such as whether there should be escalation of treatment in case of deterioration.

The convalescence hospital had a palliative team with two hospice wards. It enabled medical officers to acquire more up-to-dated knowledge in symptom relief for the dying patients and other healthcare staff to have more opportunities for receiving training in EOL care. Moreover, EOL and palliative care approach were often promoted at clinical seminars and case presentations. All of these initiatives made a significant impact on patient care in this study. In addition, the independent review on Liverpool Care Pathway (LCP) in the United Kingdom (UK) pointed out that a care pathway itself would not guarantee a quality palliative care. It only worked well if operated by welltrained, well-resourced, and sensitive clinical teams. Adequate training, education and resources should be offered to the frontline clinical staff on top of provision of guidelines with a view to increasing their motivation and self-efficacy in adopting the LCP, as well as enhancing the communication with the patients and their carers. ${ }^{16-18}$ Moreover, the attending physicians should document clearly the decision and treatment plan in advance, after discussing with patients and/or their family members, so the on-call colleagues were able to deliver a consistent care.

In Hong Kong, the law (Ambulance Ordinance) dictates that patients with cardiorespiratory arrest in the community must be resuscitated. CPR was therefore initiated in six patients, who were found to be in cardiorespiratory arrest, by the ambulance crew. To avoid this futile life-sustaining procedure, it was of paramount importance to enhance the communication between the hospitals and the ambulance service on the EOL care program through the use of Advanced Care Planning (ACP) forms. These documents would allow the patients or their proxies to clearly indicate the wish of life-sustaining treatment, such as intubation and CPR, in case of terminal illnesses, persistent vegetative state or in a state of irreversible coma. Moreover, the Ambulance Ordinance should be reviewed and amended, so that the DNR order for dying patients with an ACP could be respected by the ambulance crew. Professional development program in ACP for healthcare staff would be in place to promote their uptake of ACP. ${ }^{18}$ In addition, a detailed and clear documentation in the corporate electronic patient record about the EOL care plan could facilitate a better communication among healthcare providers even if the patients were admitted to different hospitals in Hong Kong.

\section{LIMITATIONS}

This study has several limitations. Firstly, in view of the retrospective nature of this study, some clinical information could not be retrieved from the case records, so the reasons for non-compliance were not fully evaluated in this study. Secondly, the quality indicators adopted in this study were modified from the LCP, which was strongly recommended for reform by an independent panel in 2013. ${ }^{17}$ It was revealed that the LCP could not perfectly fit into each of the cases, and there was a risk of over-emphasis on the protocol instead of the outcome of EOL care. In fact, the quality indicators we adopted were found to be capable of reviewing how the EOL care services provided rather than its outcome. Measurement of outcomes by using the questionnaires filled out by clinical staff and the family carer should be included in further studies to enable a more comprehensive assessment on the quality of EOL care. ${ }^{19}$ Thirdly, it was difficult to justify whether palliative medications on patients were "essential" in a retrospective review, as fair justification could not be made simply based on WHO recommended drug list. It should be considered with individual patient clinical context. Lastly, this was a small sample size in a local hospital in Hong Kong, so the results may not easily be generalizable to other populations.

\section{CONCLUSION}

We found significant differences in the quality of EOL care for older people with non-cancer diagnoses between an acute hospital and a convalescence hospital in Hong Kong. Greater emphasis on specialist training and education, as well as allocation of resources is needed to improve EOL care. Since the completion of this study, our services have been redesigned to improve the EOL care for older people with non-cancer diagnoses at both hospitals.

\section{ETHICAL APPROVAL}

Our study has the necessary ethical approval. The approval reference is CUHK-NTEC CRE 2015.492.s.

\section{CONFLICTS OF INTERESTS}

The authors declare that they have no conflicts of interest.

\section{REFERENCES}

1. World Health Organization, Worldwide Palliative Care Alliance. Global atlas of palliative care at the end of life. Cancer. Available at: http://www.who.int/cancer/publications/palliativecare-atlas/en/ 2014; Accessed 2015.

2. Gardiner C, Cobb M, Gott M. Barriers to providing palliative care for older people in acute hospitals. Age and Aging. 2011; 40: 233-238. doi: 10.1093/ageing/afq172

3. Reynolds K, Henderson M, Schulman A, Hanson LC. Needs of the dying in nursing homes. J Palliat Med. 2002; 5(6): 895901. doi: 10.1089/10966210260499087

4. Luk JK, Liu A, Ng WC, Lui B, Beh P, Chan FH. End-of-life care: towards a more dignified dying process in residential care homes for the elderly. Hong Kong Med J. 2010; 16(3): 235-236. 
5. Mitchell SL, Teno JM, Kiely DK, et al. The clinical course of advanced dementia. $N$ Engl J Med. 2009; 361(16): 1529-1538. doi: 10.1056/NEJMoa0902234

6. World Health Organization Cancer Program. Definition of palliative care. Available at: http://www.who.int/cancer/palliative/definition/en/ 2015; Accessed July 8, 2015.

7. Woo J. Quality of dying. In: Woo J, ed. Ageing in Hong Kong: a comparative perspective. New York, NY, USA; 2013: 235-256.

8. Tsim S, Davidson S. End-of-life care in a general respiratory ward in the United Kingdom. Am J Hosp Palliat Care. 2014; 31(2): 172-174. doi: 10.1177/1049909113481261

9. Siu WK, Liu KY, Cheung KW, et al. End-of-life-care for Chinese patients in acute care ward setting: experience in an oncology ward and report on a pilot project on the use of an integrated care pathway. Palliat Med. 2011; 25(6): 664-665. doi: $10.1177 / 0269216311407103$

10. Woo J, Lo R, Cheng JO, Wong F, Mak B. Quality of end-of-life care for non-cancer patients in a non-acute hospital. J Clin Nurs. 2011; 20: 1834-1841. doi: 10.1111/j.1365-2702.2010.03673.x

11. Connors AF, Dawson NV, Desbiens NA, et al. A controlled trial to improve care for seriously ill hospitalized patients: the study to understand prognoses and preferences for outcomes and risks of treatments (SUPPORT). JAMA.1995; 274(20): 15911598. doi: 10.1001/jama.1995.03530200027032

12. Biola H, Sloane PD, Williams CS, Daaleman TP, Zimmerman S. Preferences versus practice: life-sustaining treatments in last months of life in long term care. J Am Med Dir Assoc. 2010; 11(1): 42-51. doi: 10.1016/j.jamda.2009.07.005

13. World Health Organization. Essential medicines in palliative care: executive summary. Website: http://www.who. int/selection_medicines/committees/expert/19/applications/ PalliativeCare_8_A_R.pdf 2013; Accessed July 8, 2015.

14. Lo SH, Chan CY, Chan $\mathrm{CH}$, et al. The implementation of an end-of-life integrated care pathway in a Chinese population. Int J Palliat Nurs. 2009; 15(8): 384-388.

15. Phillips JL, Halcomb EJ, Davidson PM. End-of-Life care pathways in acute and hospice care: an integrative review. J Pain Symptom Manage. 2011; 41: 940-955. doi: 10.1016/j.jpainsymman.2010.07.020

16. McConnell T, O’Halloran P, Porter S, Donnelly M. Systematic realist review of key factors affecting the successful implementation and sustainability of the liverpool care pathway for the dying patient. Worldviews Evid Based Nurs. 2013; 10(4): 218-237. doi: 10.1111/wvn.12003
17. Department of Health. Independent report review of liverpool care pathway for dying patients. more care, less pathway: a review of the liverpool care pathway. Website: https://www. gov.uk/government/uploads/system/uploads/attachment_data/ file/212450/Liverpool_Care_Pathway.pdf 2013; Accessed July $8,2015$.

18. Samara J, Larkin D, Chan CW, Lopez V. Advance care planning in the oncology settings. Int J Evid Based Healthc. 2013; 11(2): 110-114. doi: 10.1111/1744-1609.12011

19. Verhofstede R, Smets T, Cohen J, Costantini M, Noortgate NVD, Deliens L. Improving end-of-life care in acute geriatric hospital wards using the care program for the last days of life: study protocol for a phase 3 cluster randomized controlled trial. BMC Geriatrics. 2015; 15: 13. doi: 10.1186/s12877-015-0010-7 